\title{
Effects of plasmapheresis on the course of experimental allergic neuritis in rabbits
}

\author{
JAYNE H ANTONY, JD POLlARD, JG MCLEOD \\ From the Department of Medicine, University of Sydney, Sydney, NSW, Australia
}

SUMMARY Experimental allergic neuritis was induced in 30 rabbits with extract of bovine peripheral nerve in complete Freund's adjuvant. Of the 22 animals that completed the study, nine animals were plasmapheresed within two weeks of inoculation and 13 animals served as controls. The plasmapheresed animals developed a less severe form of EAN than the controls. Differences were apparent in clinical weakness, weight loss, degree of dispersion of the muscle action potential and histological changes.

In 1955, Waksman and Adams ${ }^{1}$ induced experimental allergic neuritis(EAN) in rabbits by the subcutaneous injection of homologous or heterologous peripheral nerve in adjuvant. The same condition has subsequently been produced in other experimental animals. The clinical course of EAN and the pathological changes in peripheral nerves and roots are very similar to those in the naturally occurring human disease, acute idiopathic polyneuropathy (AIP, Guillain-Barré syndrome). ${ }^{2}$ The finding of cellular infiltrates in association with demyelinated nerves in both conditions led most investigators to conclude that pathological changes were secondary to a cell mediated autoimmune reaction against peripheral nerve myelin..$^{3-5}$ Studies on cellular immune function in vitro on patients with AIP supported this conclusion. ${ }^{6} 7$ Antimyelin activity had also been demonstrated in EAN and AIP serum in early studies but the activity in the serum correlated poorly with clinical severity of the diseases. ${ }^{189}$ In humans, serum antimyelin activity has been observed occasionally in normal people and in patients with other diseases, both neurological and non-neurological. ${ }^{10} 11$

In vivo demyelination of rat sciatic nerve by injection of EAN serum directly into the nerve was first reported by Saida and co-workers in 1977.12 This finding reopened the question of the role of humoral immunity in autoimmune peripheral demyelination. In addition, several reports have been

Address for reprint requests: Dr JH Antony, Department of Medicine, The University of Sydney, Sydney, NSW, Australia.

Received 13 August 1981

Accepted 1 September 1981 published more recently describing the benefits of plasmapheresis on the recovery of patients with AIP, chronic relapsing polyneuropathy and chronic progressive polyneuropathy. ${ }^{13-20}$ In these reports dramatic recovery was described in some patients following plasmapheresis. However, the procedure is not without risks, ${ }^{21}$ and the natural history of AIP is such that controlled trials will be necessary to $\frac{\sim}{\mathbb{D}}$ prove the efficacy of plasmapheresis in this con- $\AA$ dition. In the present study, plasmapheresis has been performed in rabbits with EAN in order to determine if it was effective in preventing the development of severe paralysis.

\section{Materials and methods}

Thirty rabbits of mixed strains weighing from $2 \cdot 2$ to $4 \cdot 1$ $\mathrm{kg}$ were used in the experiment. They were kept in wire cages and given a standard diet and water ad libitum. All of the rabbits were aged three months or more. They were randomly divided into two groups, one of which was a control group (14 animals) and the other group was plasmapheresed (16 animals). Bovine peripheral nerve or root was obtained from a local abbatoir. After the fat and connective tissue were carefully removed from the nerve, it was frozen in liquid nitrogen and crushed to a fine powder. Normal saline $(10 \mathrm{ml})$ was added to $10 \mathrm{mg}$ of crushed nerve and suspended in $20 \mathrm{ml}$ of Freund's complete adjuvant. On Day 0, each of the rabbits was anaesthetised with $100-150 \mathrm{mg}$ of intravenous propanidid (Epontal) and injected subcutaneously with $3 \mathrm{ml}$ of the above suspension. This dose was divided between the foot pads $(0.25 \mathrm{ml})$ and four separate sites on the back $(0.5 \mathrm{ml})$.

Technique of plasmapheresis

From Days 6 to 8, plasmapheresis was performed once on every animal in the plasmapheresed group. The 
animals were restrained and sedated. A disposable, winged, 21 gauge infusion needle was inserted into one of the peripheral ear veins and a vinyl catheter sutured into a central ear artery. Both arterial and venous lines were attached to a 3-way stop-cosk and kept patent by the injection of heparinised saline (1 ml of 1:5000 heparin in $100 \mathrm{ml}$ of normal saline). Plasmapheresis was carried out by the removal of either $1 / 4$ or $1 / 6$ of the rabbit's blood volume $(60 \mathrm{mg} / \mathrm{kg})$ through the arterial catheter. The blood was added to $1 \mathrm{ml}$ of $1: 5000$ heparin and centrifuged at $2000 \mathrm{~g}$ for five minutes. The plasma was removed and the cells were reinjected through the venous catheter. The procedure was repeated so that during one session a total of either $1 / 2$ or $1 / 3$ of the blood volume was removed. Some of the animals were given $10 \%$ calcium gluconate $(1 \mathrm{ml} / \mathrm{kg})$ and in the later groups oxygen was given during the procedure. Usually, no fluid replacement was used although some rabbits were given $20 \mathrm{ml}$ of normal saline intravenously at the end of the procedure. After the session the artery was tied off proximal to the tip of the catheter and the catheter removed. The duration of the procedure was 1-1 $\frac{1}{2}$ hrs. At Days 13 to 15 , a second plasmapheresis was carried out exactly as described above using the other ear. For technical reasons usually only two plasmaphereses were possible although one animal had three and another had four plasmaphereses at weekly intervals. Technical problems arose when trying to insert a catheter proximal to the previous catheter site since the artery had usually thrombosed.

The animals were weighed at the beginning of the experiment and then at approximately weekly intervals thereafter. Some of the plasmapheresed animals were also weighed before and after the procedure. Clinical evaluation was also carried out at weekly intervals by two independent observers. The following clinical grading was used: $0-$ No weakness, $1-$ Hindlegs possibly weak, 2-Hindlegs trembling and ataxic, 3-Hindlegs severely weak; unable or barely able to use, 4- Hind and forelegs very weak.

\section{Electrophysiological techniques}

In the fifth week, neurophysiological studies were performed after inducing anaesthesia with $100-150 \mathrm{mg}$ intravenous propanidid followed by a mixture of halothane and oxygen by face mask. Motor conduction velocity was recorded in both sciatic nerves of each animal. The compound action potential was recorded from the small muscles of the foot through a pair of subcutaneous steel needle electrodes; the proximal recording electrode was inserted into the muscle mass and a remote electrode was inserted distally into one of the toes of the same foot. The nerve was stimulated percutaneously through a pair of needle electrodes separated by about $1 \mathrm{~cm}$ at the level of both the ankle and the sciatic notch. The stimulus was a square wave of duration $0.2 \mathrm{~ms}$. derived from a Devices stimulator. Action potentials were amplified, displayed on an oscilloscope and photographic recordings made on polaroid film. To prevent heat loss during recordings the animals were placed beneath a lamp. The temperature of the limb was measured with a thermistor at each examination and ranged from $34^{\circ}$ to $37^{\circ} \mathrm{C}$.

\section{Histological techniques}

Immediately after completion of the neurophysiological studies, the animals were anaesthetised with $60-120 \mathrm{mg}$ of sodium pentobarbitone and perfused with $4 \%$ glutaraldehyde through the abdominal aorta. After perfusion, $1 \mathrm{~cm}$ segments of both sciatic nerves were removed immediately distal to the sciatic notch and similar lengths of 4th and 5th lumbar dorsal and ventral roots were also removed in some animals. Nerve segments were placed in $3 \%$ glutaraldehyde in a $0.1 \mathrm{M}$ cacodylate buffer for $3 \mathrm{hrs}$ followed by $2 \%$ osmium tetroxide for $1 \mathrm{hr}$. Specimens were dehydrated in graded concentrations of alcohol and embedded in epoxy resin (Spurr's). Thick sections $(1 \mu \mathrm{m})$ were stained with toluidine blue and prepared for light microscopy.

\section{Results}

COMPLICATIONS OF PLASMAPHERESIS

Of the 16 animals in the plasmapheresed group, six died as a result of the procedure. Five animals died at $7,8,12,19$ and 21 days respectively during the procedure. None was weak at the time and only one had weight loss. Four of those five animals became restless, began to hyperventilate and assumed an opisthotonic posture immediately prior to death. The fifth animal had a chest infection prior to the procedure and died suddenly while being plasmapheresed. A hypoxic insult was suspected in all cases. The animals in the later groups were given oxygen during the procedure and this improved their survival and well-being. No postmortem studies were performed. The sixth death occured $24 \mathrm{hrs}$ after the first plasmapheresis. The animal was noted to be well at $20 \mathrm{hrs}$, but shortly thereafter, began falling to the right and was unable to walk. Because of its deteriorating condition, it was killed.

\section{CLINICAL ASSESSMENT}

The average weight of both plasmapheresed and control groups at the beginning of the experiment was 3.3 kilograms. The percentage change in body weight from Day 0 to $2-3$ weeks was $-3 \%$ (SD, $10 \%$ ) in the control animals compared to $-2 \%(\mathrm{SD}, 7 \%)$ in those animals that were plasmapheresed, an insignificant difference. However, from 2-3 weeks to the fifth week, the control animals had a further change in weight of $-8 \%(\mathrm{SD}, 7 \%)$ compared to $-2 \%(\mathrm{SD}, 5 \%)$ in the plasmapheresed group. The difference was significant $(p<0.05)$.

There were also differences on clinical assessment. When examined at 2-3 weeks, five of the 14 control animals had definite weakness whereas none of the plasmapheresed animals was weak. At five weeks, 11 of the surviving 13 control animals had definite and often marked weakness, but only three of the nine plasmapheresed animals were definitely weak (table 1). Two animals, one in the control group and 
Table 1 Clinical assessment of animals

\begin{tabular}{|c|c|c|}
\hline \multirow[t]{2}{*}{ Animal } & \multicolumn{2}{|c|}{ Grade of weakness } \\
\hline & 2-3 weeks & 5 weeks \\
\hline \multicolumn{3}{|c|}{ Control group } \\
\hline $\mathrm{ZCl}$ & 0 & 2 \\
\hline $\mathrm{ZC2}$ & 0 & 2 \\
\hline $\mathrm{ZC} 3$ & 1 & - \\
\hline $\mathrm{ACl}$ & 2 & 2 \\
\hline $\mathrm{AC} 2$ & 2 & 1 \\
\hline $\mathrm{AC} 3$ & 1 & 1 \\
\hline $\mathrm{AC} 4$ & 1 & 3 \\
\hline AC5 & 2 & 3 \\
\hline YC1 & 2 & 3 \\
\hline YC2 & 1 & 2 \\
\hline YC3 & 2 & 3 \\
\hline $\mathrm{BCl}$ & 1 & 2 \\
\hline BC2 & 1 & 3 \\
\hline $\mathrm{BC} 3$ & 0 & 2 \\
\hline \multicolumn{3}{|c|}{ Plasmapheresed group } \\
\hline ZPI & 0 & 2 \\
\hline ZP3 & 0 & - \\
\hline AP1 & 1 & 1 \\
\hline AP2 & 0 & 1 \\
\hline AP3 & 0 & - \\
\hline AP4 & 0 & 1 \\
\hline YP1 & 0 & 2 \\
\hline YP2 & 0 & 2 \\
\hline YP3 & 1 & 1 \\
\hline BP2 & 0 & 0 \\
\hline BP4 & 1 & - \\
\hline BP5 & 0 & 1 \\
\hline
\end{tabular}

one in the plasmapheresed group, died in the third week after developing an early fulminating form of EAN.

\section{NEUROPHYSIOLOGICAL STUDIES}

There were no significant differences in the distal motor latency, or conduction velocities of the sciatic nerves or in the amplitude of the muscle action potentials in the two groups that were studied in the fifth week (table 2). Dispersion was more evident in the control animals (fig 1) and there was a significant difference in the duration of the action potentials between the controls and plasmapheresed animals. In the control animals 18 of 26 hindlimbs had dispersed muscle potentials while six of the 18 hindlimb muscle action potentials were dispersed in the plasmapheresed animals.

\section{PATHOLOGY}

The sciatic nerves of all the 22 animals in the control and plasmapheresed group that survived to five
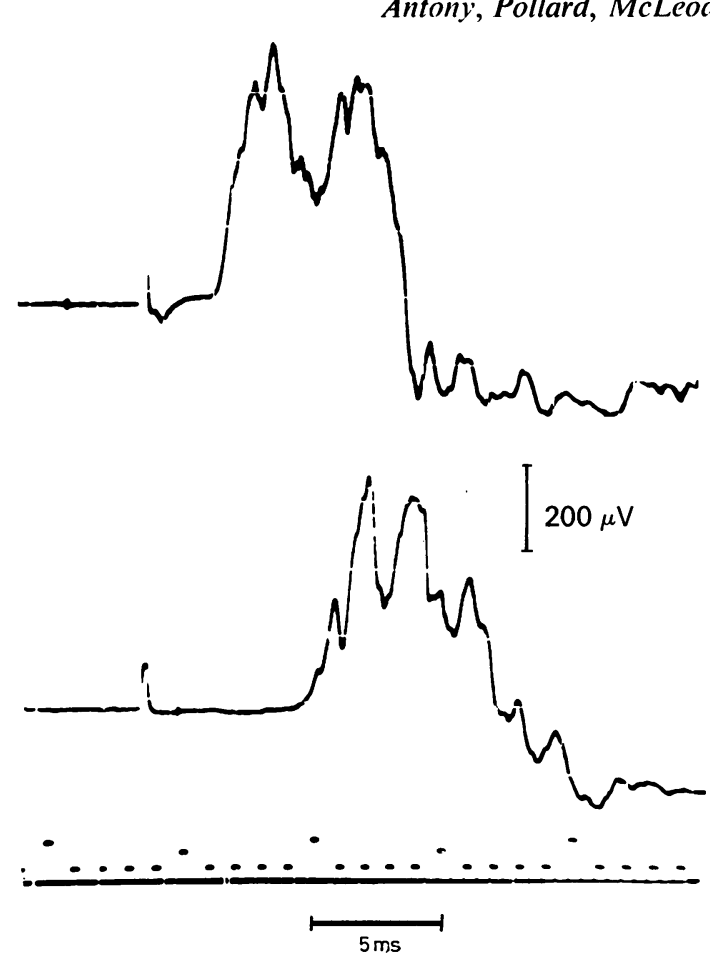

Fig 1 Dispersed muscle action potential recorded from foot muscle of a control animal on stimulating at ankle (above) and sciatic notch (below).

weeks were removed for analysis after perfusion. In addition, lumbar roots were removed in 13 animals. The pathological studies confirmed the observation that the control animals were more affected by EAN than the plasmapheresed animals. No definite changes of demyelination were seen on light microscopy in any of the plasmapheresed animals. By contrast, in the control group there were severe changes in two animals (fig 2), mild changes in eight and no abnormalities were seen in three animals.

\section{Discussion}

No studies of the effects of plasmapheresis on the course of EAN have been published previously. In the present study, plasmapheresis was performed in the first and second week after sensitisation of the

Table 2 Neurophysiological studies of sciatic nerves at five weeks

\begin{tabular}{|c|c|c|c|}
\hline & Control group & Plasmapheresed group & Significant differences* \\
\hline $\begin{array}{l}\text { Latency } \\
\text { Conduction velocity } \\
\text { Amplitude } \\
\text { Duration action potential }\end{array}$ & $\begin{array}{r}2.6 \pm 0.7 \mathrm{~ms}(\mathrm{~N}=25) \\
59.0 \pm 9.0 \mathrm{~ms}(\mathrm{~N}=24) \\
5.3 \pm 4.4 \mathrm{mv}(\mathrm{N}=23) \\
8.6 \pm 4.1 \quad(\mathrm{~N}=26)\end{array}$ & $\begin{array}{r}2.5 \pm 0.5 \mathrm{~ms}(\mathrm{~N}=17) \\
63.0 \pm 5.0 \mathrm{~ms}(\mathrm{~N}=18) \\
7 \cdot 8 \pm 7 \cdot 3 \quad(\mathrm{~N}=16) \\
6 \cdot 3 \pm 2.0 \quad(\mathrm{~N}=18)\end{array}$ & $\begin{array}{l}\text { NS } \\
\text { NS } \\
\text { NS } \\
\mathrm{p}<0.05\end{array}$ \\
\hline
\end{tabular}

(*Student $\mathrm{t}$ test)

(NS = not significant $)$ 


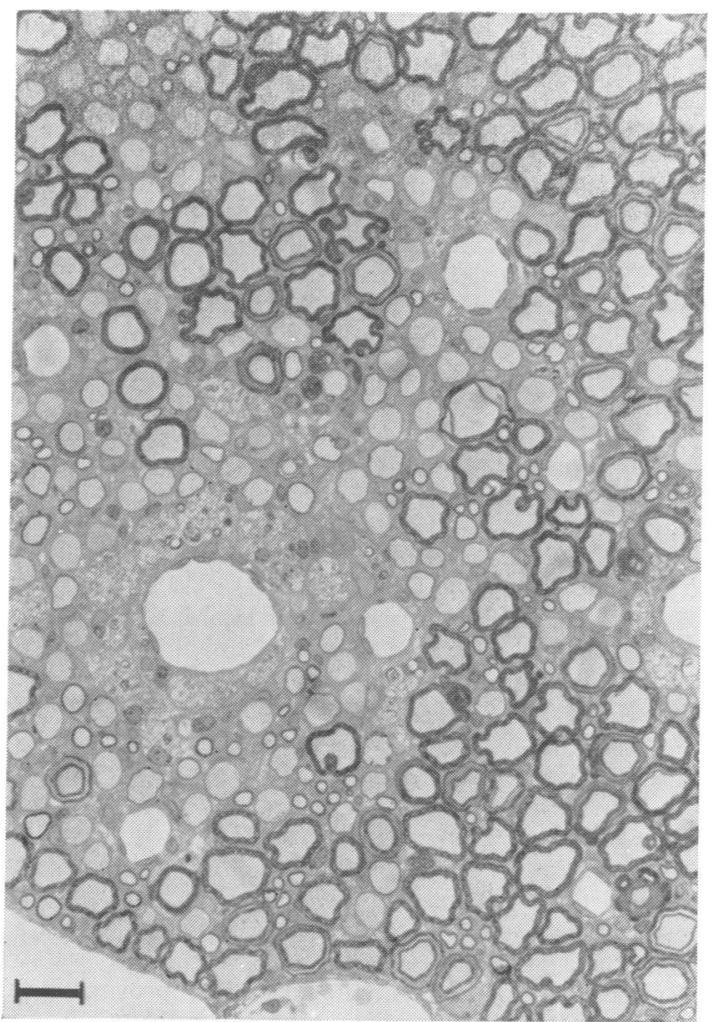

Fig 2 Photomicrograph of ventral root of a control animal showing perivenous demylination with thinly myelinated and bare axons with cellular infiltration (Toluidine blue) Bar $=8 \mu$.

rabbits with bovine peripheral nerve before any clinical illness appeared. Although this obviously would not correlate with plasmapheresis as used in human diseases, we considered it was important to study the effects of plasmapheresis upon the development of the disease. Moreover, the severe technical limitations of this procedure in animals do not allow the intensive exchange programme which is used in man, and we therefore considered marginal benefits might be more easily seen by this approach. Our results indicate that EAN is modified by the plasmapheresis of $1 / 2$ to $1 / 3$ the blood volume weekly in the first two weeks after sensitisation with bovine peripheral nerve. Since we perfused all the animals at five weeks we cannot be certain that the plasmapheresed group would not have developed EAN at a later date or else developed a more chronic form. Traugott and Raine ${ }^{22}$ described a more chronic form of experimental allergic encephalomyelitis in adult guinea pigs that developed after frequent withdrawals of blood samples.
Control animals lost more weight than the animals that were plasmapheresed. This is an important finding since weight loss is a well described feature of EAN. Lack of weight loss in the plasmapheresed group was not related to fluid overload since the only fluid returned to the animals was $20 \mathrm{ml}$ of normal saline. In addition, several of the animals were weighed before and after plasmapheresis and the weight was stable. Clinical assessment of the animals showed differences between the groups when assessed blindly by two different observers.

In the neurophysiological studies at five weeks no differences between plasmapheresed and control groups in distal motor latency or conduction velocity of the sciatic nerves were found. This is not surprising since previous experimental work has shown that considerable demyelination can be present in animals with little change in conduction velocity. ${ }^{23}$ However, dispersion of the action potential, which is a more reliable indicator of $^{f}$ the degree of demyclination, was more marked in the control group. In the pathological studies of the sciatic nerve and lumbar nerve roots more demyelination was observed in the control group than in the plasmapheresed group which showed no definite areas of demyelination in the sections that were studied. Since some of the plasmapheresed animals were weak, demyelination was certainly present. The disease is patchy, and it is possible that we found more lesions in the control group by chance since more areas were affected than in the nerves of the plasmapheresed group.

The method of plasmapheresis used in this study was not very satisfactory because of the high mortality rate directly related to the procedure. Only one description of a method of plasmapheresis of experimental animals has appeared in the literature. ${ }^{24}$ This method was similar in principle to ours except that a scalp vein needle was directly inserted into the central ear artery of the rabbit. When we tried this method the needle caused vasoconstriction and we were unable to plasmapherese the volumes described in the report. The other factor contributing to the high mortality rate may have been the short time ( 1 to $2 \mathrm{hrs)}$ taken for plasmapheresis. This may have been too fast for some rabbits, but it was difficult to restrain the animals for longer periods. The other disadvantage of our technique was the number of possible plasma exchanges which was usually limited to two because of thrombosis of ear arteries. Despite the technical problems encountered in plasmapheresis of rabbits, the present study has demonstrated that prophylactic plasma exchange mitigates the severity of the weakness and the degree of demyelination caused by EAN. These findings lend support to the work of Saida et al ${ }^{12}$ that suggest 
that a serum factor is important in the pathogenesis of the disease. Further studies are in progress to assess the value of plasmapheresis in influencing the course of the illness in animals after EAN is clinically established, and to characterise serum factors responsible for demyelination in EAN.

\section{References}

1 Waksman BH, Adams RD. Allergic neuritis: an experimental disease of rabbits induced by the injection of peripheral nervous tissue and adjuvants. $J$ Exp Med 1955;102:213-36.

${ }^{2}$ Arnason BGW. Idiopathic polyneuritis (LandryGuillain-Barré syndrome) and experimental allergic neuritis. A comparison. In: Rowland L, ed. Immunological Disorders of the Nervous System. Baltimore: Williams and Wilkins Res Publ Assoc for Res in Nerve and Mental Diseases, 1971 ;49:156-77.

${ }^{3}$ Wiśniewski H, Terry RD, Whitaker JN, Cook SD, Dowling PC. Landry-Guillain-Barré syndrome. A primary demyelinating disease. Arch Neurol 1969; $21: 269-76$.

4 Prineas JW. Acute idiopathic polyneuritis. An electron microscope study. Lab Invest 1972;26:133-47.

${ }^{5}$ Lampert PW. Mechanisms of demyelination in experimental allergic neuritis. Electron microscopic studies. Lab Invest 1969;20:127-38.

${ }^{6}$ Abramsky O, Webb C, Teitelbaum D. Cell-mediated immunity to neural antigens in idiopathic polyneuritis and myeloradiculitis. Clinical immunological classification of several autoimmune demyelinating disorders. Neurology (Minneap) 1975;25:1154-9.

${ }^{7}$ Sheremata W, Colby S, Lusky G, Cosgrove JBR. Cellular hypersensitisation to peripheral nervous antigens in the Guillain-Barré syndrome. Neurology (Minneap) 1975;25:833-9.

${ }^{8}$ Caspary EA, Field EJ. Antibody response to central and peripheral nerve antigens in rat and guinea pig. J Neurol Neurosurg Psychiatry 1965;28:179-82.

${ }^{9}$ Cook SD, Dowling PC, Murray MR, Whitaker JN. Circulating demyelinating factors in acute idiopathic polyneuropathy. Arch Neurol 1971 ;24:136-44.

${ }^{10}$ Melnick SC. Thirty-eight cases of the Guillain-Barré syndrome: an immunological study. Brit Med $J$ $1963 ; 1: 368-73$.

11 Tse KS, Arbesman CE, Tomasi TB, Tourville D. Demonstration of antimyelin antibodies by immuno- fluorescence in Guillain-Barré syndrome. Clin Exp Immunol $1971 ; 8: 881-7$.

12 Saida T, Saida K, Silberberg DH, Brown MJ. Transfer of demyelination by intraneural injection of experimental allergic neuritis serum. Nature 1978; 272:639-41.

${ }^{13}$ Brettle RP, Gross M, Legg NJ, Lockwood M, Pallis C. Treatment of acute polyneuropathy by plasma exchange. Lancet 1978;11:1100.

${ }^{14}$ Fowler H, Volpe M, Marks G, Egolf C, Dau PC. Recovery from chronic progressive polyneuropathy after treatment with plasma exchange and cyclophosphamide. Lancet 1979;11:1193.

${ }^{15}$ Levy RL, Newkirk R, Ochoa J. Treating chronic relapsing Guillain-Barré syndrome by plasma exchange. Lancet $1979 ; 1: 259-60$.

${ }^{16}$ Mark B, Hurwitz BJ, Olanow CW, Foy JW. Plasmapheresis in idiopathic inflammatory polyradiculoneuropathy. Neurology (Minneap) 1980;30:361.

17 Server AC, Lefkowith J, Braine H, McKhann GM. Treatment of chronic relapsing inflammatory polyradiculoneuropathy by plasma exchange. $A n n$ Neurol 1979;6:258-61.

${ }^{18}$ Toyka KV, Augspash R, Paulus W, Grabensee B, Hein D. Plasma exchange in polyradiculoneuropathy. Ann Neurol 1980;8:205-6.

19 Toyka KV, Augspach R, Besinger UA, Grabensee B. Treatment of myasthenia gravis and Guillain-Barré syndrome with plasma exchange. In: Sieberth HG and Schattauer FK, eds. Plasma Exchange. Stuttgart: Verlag, 1980:290-6.

${ }^{20}$ Ropper AH, Shahani B, Huggins CE. Improvement in four patients with acute Guillain-Barré syndrome after plasma exchange. Neurology (Minneap) 1980; 30:361.

${ }^{21}$ Brewer EJ, Nickeson RW, Rossen RD. Plasma exchange in selected patients with juvenile rheumatoid arthritis. J Peds $1981 ; 98: 190-4$.

22 Traugott U, Raine CS. EAE-Frequent sampling of blood alters disease course. Acta Neuropath 1979; 48:171-5.

${ }^{23}$ Hall JI. Studies of demyelinated peripheral nerves in guinea pigs with experimental allergic neuritis. A histological and electrophysiological study. Part II Electrophysiological observations. Brain 1967;90: 313-32.

21 Wohl H, Weekly L. High volume antisera yields from rabbits using plasmapheresis. J Immunol Methods 1979;27:97-9. 